\title{
Diversidad de musgos en el estado de Aguascalientes, MÉXICO
}

\author{
Claudio Delgadillo-Moya ${ }^{1}$, José luis Villaseñor, Enrique Ortiz y Ana Paola Peña-Retes \\ Universidad Nacional Autónoma de México, Departamento de Botánica, Instituto de Biología, \\ Apartado Postal 70-233, 04510 México, D.F. México. \\ 1'Autor para la correspondencia: moya@unam.mx
}

\begin{abstract}
Resumen: La exploración del estado de Aguascalientes documenta la existencia de 45 especies y variedades en la flora de musgos incluyendo siete especies citadas previamente en la literatura. Se supone que las especies registradas representan una extensión de la floras de las zonas secas de Jalisco y Zacatecas. Aunque se requiere mayor exploración de campo, los análisis de riqueza florística y distribución potencial estiman que la flora del estado es comparativamente pequeña (91 especies). Los sitios más altos en el occidente del estado, principalmente, deberán explorarse para conocer la diversidad de su flora de musgos.
\end{abstract}

Palabras clave: distribución potencial, Jalisco, modelaje, riqueza florística, Zacatecas.

\begin{abstract}
Current exploration documents the presence of 45 species and varieties in the moss flora of the state of Aguascalientes, including seven taxa previously cited in the literature. These species seem to represent an extension of the dry land flora of the states of Jalisco and Zacatecas. Even though additional fieldwork is required, floristic richness and potential distribution analyses indicate that the state moss flora is comparatively small in number with at least 91 species. At least, the higher sites in the western part of Aguascalientes should be explored to obtain a better idea of the diversity of its moss flora.
\end{abstract}

Key words: floristic richness, Jalisco, modeling, potential distribution, Zacatecas.

L a exploración de las zonas montañosas del centro y sur de México ha sido motivo de numerosos esfuerzos de colectores y briólogos profesionales que han producido colecciones, publicaciones y datos florísticos para el país. En contraste, los estados del centro, al sur de la Altiplanicie Mexicana, han sido poco explorados y su flora de musgos es poco conocida. Hasta antes de 1979, la información sobre los musgos del estado de Zacatecas incluía solamente siete especies (Delgadillo-M y Cárdenas-S, 1979); después de los primeros estudios formales (Delgadillo-M y Cárdenas-S, 1987), el número se elevó a 115 especies y variedades. De manera similar, para Guanajuato se conocían unos 20 registros, pero los trabajos preliminares mostraron que había, cuando menos 114 especies y variedades (Delgadillo y Cárdenas, 1996). Por su parte, Herrera-Paniagua et al. (2008) hicieron notar que sólo se conocían cuatro especies en Querétaro antes de que Sharp et al. (1994) citaran 44; actualmente ese número supera las 200 especies de musgos.

La publicación de la flora de musgos de México (Sharp et al., 1994) actualizó la información sobre nuestras espe- cies, pero no resolvió el precario conocimiento para varias entidades del país, incluyendo el estado de Aguascalientes. La revisión de esa obra y del listado de Crum (1951) indica que sólo hay siete especies de musgos formalmente registradas para esa entidad. Por este motivo y para contar con datos comparativos biogeográficos y de diversidad, en esta contribución presentamos los resultados de los trabajos de campo en el estado y las estimaciones sobre la riqueza de su flora de musgos, así como predicciones de su distribución potencial.

\section{Materiales y métodos}

Entre el 27 de agosto y el 1 de septiembre de 2013, se hicieron recorridos en 15 localidades en los municipios de El Llano, Tepezala, Jesús María, Calvillo, San José de Gracia y Rincón de Romos del estado de Aguascalientes donde se obtuvieron 109 ejemplares. Las coordenadas geográficas de cada localidad se muestran en el Tabla 1. Estas colecciones provienen principalmente de matorrales en el sur y sureste 
Tabla 1. Ubicación de las localidades de muestreo de musgos en Aguascalientes. Los números marcados con P.P. fueron colectados por Paola Peña; los de cuatro dígitos corresponden a las colecciones de C. Delgadillo.

\begin{tabular}{|c|c|c|c|}
\hline Números & Localidad & Coordenadas $(\mathrm{N}-\mathrm{O})$ & Altitud (m) \\
\hline PP248-250; 7555-7556 & 5 km NE de Palo Alto & $21^{\circ} 57^{\prime} 09^{\prime \prime}-101^{\circ} 56^{\prime} 08^{\prime \prime}$ & 2,119 \\
\hline $7557-7560$ & Est. Microondas Tepezala & $22^{\circ} 12^{\prime} 48^{\prime \prime}-102^{\circ} 08^{\prime} 12^{\prime \prime}$ & 2,320 \\
\hline PP251-252; 7561-7565 & 4 km NE de los Caños & $21^{\circ} 47^{\prime} 49^{\prime \prime}-102^{\circ} 26^{\prime} 25^{\prime \prime}$ & 1,870 \\
\hline PP253-256; 7566-7571 & $6 \mathrm{~km} \mathrm{~S} \mathrm{de} \mathrm{Tapias} \mathrm{Viejas}$ & $21^{\circ} 47^{\prime} 26^{\prime \prime}-102^{\circ} 31^{\prime} 51^{\prime \prime}$ & 1,960 \\
\hline PP257-259; 7572-7579 & Presa de los Serna & $21^{\circ} 18^{\prime} 11^{\prime \prime}-102^{\circ} 50^{\prime} 30^{\prime \prime}$ & 1,740 \\
\hline PP260-265; 7580-7594 & 1 km W cascada El Garruño & $21^{\circ} 44^{\prime} 11^{\prime \prime}-102^{\circ} 43^{\prime} 45^{\prime \prime}$ & 2,040 \\
\hline 7595-7596 & $1 \mathrm{~km}$ E cascada El Garruño & $21^{\circ} 44^{\prime} 24^{\prime \prime}-102^{\circ} 43^{\prime} 14^{\prime \prime}$ & 2,160 \\
\hline 7597-7605 & El Chiquihuite & $21^{\circ} 54^{\prime} 08^{\prime \prime}-102^{\circ} 46^{\prime} 50^{\prime \prime}$ & 1,930 \\
\hline 7606 & Cerca del Cerro El Mirador & $22^{\circ} 05^{\prime} 35^{\prime \prime}-102^{\circ} 42^{\prime} 54^{\prime \prime}$ & 2,520 \\
\hline PP266; 7607-7610 & $3 \mathrm{~km}$ al E de El Sinai & $22^{\circ} 08^{\prime} 04^{\prime \prime}-102^{\circ} 41^{\prime} 27^{\prime \prime}$ & 2,560 \\
\hline PP267-268; 7611-7613 & Paraíso Amalita & $22^{\circ} 09^{\prime} 04^{\prime \prime}-102^{\circ} 39^{\prime} 50^{\prime \prime}$ & 2,550 \\
\hline 7614-7615 & Boquillas & $22^{\circ} 15^{\prime} 18^{\prime \prime} \quad 102^{\circ} 23^{\prime} 05^{\prime \prime}$ & 2,000 \\
\hline PP269-272; 7616-7620 & $1 \mathrm{~km} \mathrm{~W}$ de Las Camas & $22^{\circ} 15^{\prime} 03^{\prime \prime}-102^{\circ} 25^{\prime} 09^{\prime \prime}$ & 2,080 \\
\hline PP273-277; 7621-7627 & Base del cerro La Campana & $21^{\circ} 57^{\prime} 23^{\prime \prime}-102^{\circ} 34^{\prime} 14^{\prime \prime}$ & 2,150 \\
\hline 7628 & El Colorín & $21^{\circ} 57^{\prime} 14^{\prime \prime}-102^{\circ} 29^{\prime} 41^{\prime \prime}$ & 2,030 \\
\hline
\end{tabular}

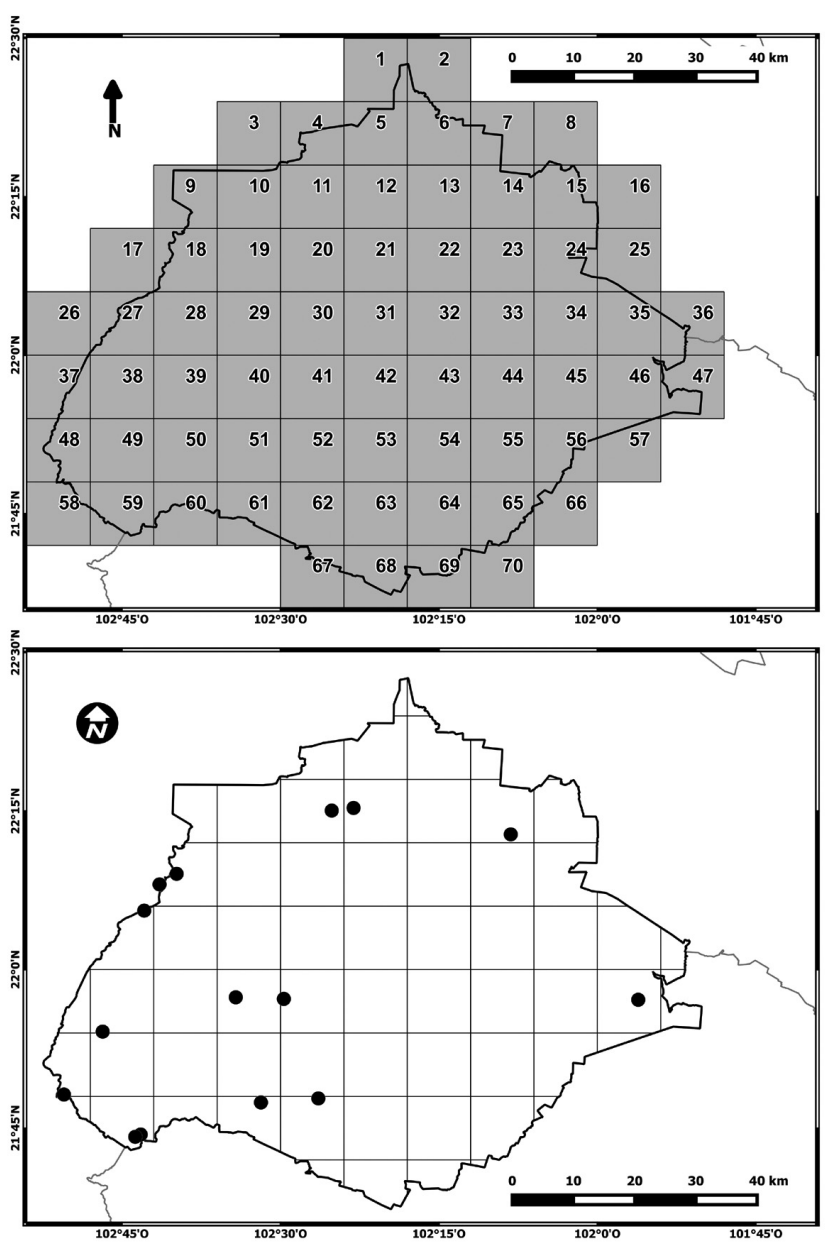

Figura 1. División del estado de Aguascalientes en celdas de 6' $\times$ 6' de latitud y longitud para llevar a cabo los análisis de diversidad ( $\mathrm{N}=70$ celdas) y localidades de recolecta de especies de musgos. En el mapa inferior, los puntos indican 15 sitios de colecta y un total de 109 registros. y de algunos encinares del este y noroeste de la entidad. Los ejemplares determinados fueron depositados en la Colección del Briofitas del Herbario Nacional (MEXU) y los duplicados disponibles se distribuyeron a otros herbarios.

La nomenclatura sigue la revisión actualizada de Latmoss 2010 (Delgadillo-Moya, 2010).

Con los datos de 109 registros georreferenciados de 40 especies de musgos de Aguascalientes, se calculó primero el tamaño de celda más adecuado para realizar los análisis posteriores. Siguiendo la recomendación de la Unión Internacional para la conservación de la Naturaleza (IUCN, 2001), se determinó el área de ocupación (AOO) de cada especie, que sugiere que el tamaño del celda tome en cuenta el $10 \%$ de la distancia del eje más largo entre dos puntos de recolecta (Suárez-Mota y Villaseñor, 2011). Para realizar dicho cálculo, se empleó la extensión "Conservation Assessment Tools" diseñada para Arcview (Moat, 2007). Para 24 especies no había registros suficientes para determinar su área de ocupación, por lo que se optó por emplear como tamaño de celda el $10 \%$ de la distancia del eje más largo en Aguascalientes; este estado mide $108 \mathrm{~km}$ de extremo a extremo, por lo que se estableció como ancho de la celda una distancia de $10.8 \mathrm{~km}$ por lado. Posteriormente, dicho ancho en kilómetros fue transformado a minutos de arco, con lo que se obtuvo una medida de 6 minutos, la cual fue empleada para formar una malla en Aguascalientes, con la que se realizaron los análisis que se mencionan en los siguientes párrafos (Figura 1).

Esfuerzo de recolecta. Mediante los datos geográficos de los registros de recolecta que se emplearon para documentar la riqueza de especies de musgos, se construyó una curva de acumulación de especies (Gotelli y Colwell, 2001). Las unidades de muestreo fueron 13 celdas de 6 minutos por lado que presentaron registros (Tabla 2). Teóricamente, la asín- 
Tabla 2. Riqueza de especies conocida y potencial en cada una de las celdas de 6 minutos por lado en que se dividió el estado de Aguascalientes. Los números de celda corresponden a los indicados en la figura 1.

\begin{tabular}{|c|c|c|}
\hline $\begin{array}{l}\text { Número de } \\
\text { especies }\end{array}$ & $\begin{array}{l}\text { Celdas con riqueza } \\
\text { conocida }\end{array}$ & $\begin{array}{l}\text { Celdas con riqueza } \\
\text { potencial }\end{array}$ \\
\hline 1 & 27,41 & 63 \\
\hline 2 & 46 & $13,22,34,43,64,65,70$ \\
\hline 3 & 12 & $44,53,54,56,66$ \\
\hline 4 & $14,38,62$ & $32,33,35,45,55,58$ \\
\hline 5 & & 6,8 \\
\hline 6 & 61 & 69 \\
\hline 7 & 48 & $2,7,12$ \\
\hline 8 & 11 & $21,24,36,49,62$ \\
\hline 9 & 40 & 42 \\
\hline 10 & & 16 \\
\hline 11 & 18,59 & $15,51,52,57$ \\
\hline 12 & & $1,5,20,23$ \\
\hline 15 & & 4,46 \\
\hline 16 & & 30 \\
\hline 17 & & $14,39,41,50$ \\
\hline 18 & & 19,26 \\
\hline 19 & & $17,29,37$ \\
\hline 20 & & 60 \\
\hline 22 & & 27 \\
\hline 23 & & 38 \\
\hline 24 & & 11,61 \\
\hline 27 & & 40,48 \\
\hline 31 & & 59 \\
\hline 32 & & 18 \\
\hline
\end{tabular}

tota de la curva de acumulación se relaciona con el número de especies que se pueden encontrar en la zona de estudio (Jiménez-Valverde y Hortal, 2003). El número de celdas se usó como medida del esfuerzo de muestreo y se ordenaron aleatoriamente 50 veces, con el fin de construir una curva suavizada empleando el programa EstimateS versión 8.2.0 (Colwell, 2009). La asíntota de la curva se estimó ajustando la ecuación de Clench a la curva de acumulación (Soberón y Llorente, 1993; Colwell y Coddington, 1994) mediante el método Simplex and Quasi-Newton en el programa STATISTICA (StatSoft, 2011).

Modelación. Los modelos de nicho ecológico de las especies son una herramienta que ha demostrado su utilidad en el llenado de los huecos u omisiones en los mapas de distribución basados en ejemplares (Newbold, 2010; Peterson et al., 2011). Los modelos identifican áreas ambientalmente similares a los sitios donde las especies ya han sido encontradas, pero donde no existen colectas. Para llenar los vacíos de información observables en la figura 2 se realizaron los modelos de nicho ecológico de las especies de musgos registradas en Jalisco y Zacatecas, pero ausentes en Aguascalientes. Se toma como supuesto que si las condiciones ambientales en que se desarrollan los musgos de Jalisco

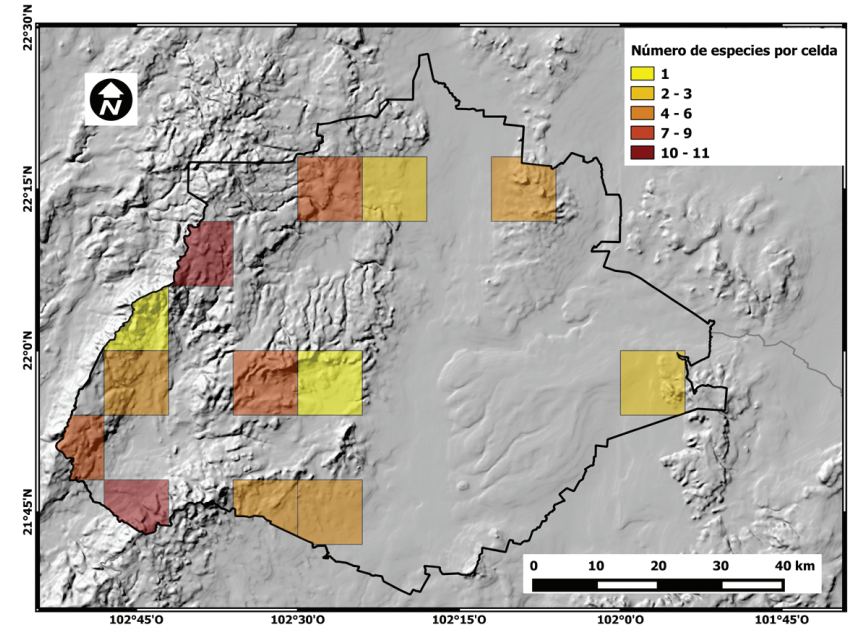

Figura 2. Riqueza conocida de los musgos en el estado de Aguascalientes $(\mathrm{N}=39$ especies). Los tonos indican el número de especies registradas en cada celda $\left(6^{\prime} \times 6^{\prime}\right)$.

y Zacatecas, se encuentran en Aguascalientes, entonces los modelos de nicho ecológico predecirán los sitios donde podríamos encontrar tales especies en este último estado.

Con los datos de herbario se obtuvieron los registros de 45 especies presentes en Jalisco y Zacatecas, pero no en Aguascalientes; solamente 41 de ellas tuvieron los registros suficientes para elaborar los modelos, con el objetivo de complementar el conocimiento de la flora briológica del estado de Aguascalientes. Para cada especie hubo de 3 a 62

Tabla 3. Variables bioclimáticas utilizadas para generar los modelos de distribución potencial (Hijmans et al., 2005; López-Mata et al., 2012).

\begin{tabular}{cl}
\hline Variables & \multicolumn{1}{c}{ Descripción } \\
\hline Bio1 & Temperatura media anual \\
Bio2 & Variación media diurna \\
Bio3 & Isotermalidad \\
Bio4 & Estacionalidad de la temperatura \\
Bio5 & Máxima temperatura del trimestre más cálido \\
Bio6 & Mínima temperatura del trimestre más frío \\
Bio7 & Variación anual de la temperatura \\
Bio8 & Media de la temperatura del cuarto trimestre más húmedo \\
Bio9 & Media de la temperatura del cuarto trimestre más seco \\
Bio10 & Media de la temperatura del cuarto trimestre más cálido \\
Bio11 & Media de la temperatura del cuarto trimestre más frío \\
Bio12 & Precipitación anual \\
Bio13 & Precipitación del trimestre más húmedo \\
Bio14 & Precipitación del trimestre más seco \\
Bio15 & Estacionalidad de la precipitación \\
Bio16 & Precipitación del cuarto trimestre más húmedo \\
Bio17 & Precipitación del cuarto trimestre más seco \\
Bio18 & Precipitación del cuarto trimestre más cálido \\
Bio19 & Precipitación de cuarto trimestre más frío \\
etra & Evapotranspiración \\
\hline
\end{tabular}


registros; cuatro especies no contaban con el número mínimo (3 registros) considerado en el presente trabajo para elaborar los modelos de nicho ecológico.

Se consideraron 20 variables como predictores potenciales (Tabla 3), cada una con una resolución de $1 \mathrm{~km}^{2}$, para los estados de Aguascalientes, Jalisco y Zacatecas. Las variables utilizadas se describen con mayor detalle en CruzCárdenas et al. (2012).

La selección de los registros empleados para entrenar el modelo se realizó en función del número de registros disponible para cada especie. En el caso de las especies con cinco o más registros, los datos fueron fraccionados en $75 \%$ para entrenar el modelo y el $25 \%$ restante para validarlo, mientras que para las especies que contaban con menos de cinco, se empleó el número total de datos $(100 \%)$ para entrenar el modelo.

Las 20 variables y los registros de entrenamiento y de validación se utilizaron para generar los modelos de nicho ecológico de las especies mediante el empleo del algoritmo Maxent, basado en el principio de máxima entropía (http:// www.cs.princeton.edu/ schapire/maxent/). Maxent requiere solamente registros de presencia de las especies y un conjunto de variables ambientales predictivas (Phillips et al., 2006). La configuración de Maxent fue por defecto (Phillips y Dudik, 2008), excepto que se indicó un umbral de corte con valor del "Maximum training sensitivity plus specificity" (Liu et al., 2013) para obtener modelos binarios. De esta manera se obtuvo un mapa con la distribución del nicho ecológico para cada una de las 41 especies.

Los datos de distribución conocidos de las 39 especies con registros fehacientes en Aguascalientes y los modelos de nicho ecológico de las 41 especies presentes en Jalisco y Zacatecas, y proyectados hacia Aguascalientes, fueron cruzados contra la malla de seis minutos para obtener el número potencial de especies por celda.

\section{Resultados}

Consideraciones florísticas. La lista de la Tabla 4 contiene un total de 45 taxa específicos o infraespecíficos para la flora de musgos del estado de Aguascalientes. De ellas, 38 son nuevos registros para esa flora, seis fueron citadas en Sharp et al. (1994), y una fue colectada por nosotros, pero había sido registrada previamente. A continuación se ofrecen algunas observaciones sobre esos taxa. La presencia de Braunia secunda debe confirmarse mediante la revisión de los ejemplares que motivaron su inclusión en Sharp et al. (1994) o a través de ejemplares del campo. Braunia secunda y $B$. andrieuxii se habían considerado como sinónimos, pero De Luna y Gómez-Velasco (2008) mostraron que había diferencias significativas por las que, por el momento, los dos nombres deben retenerse como parte del listado de especies. Grimmia elongata, G. laevigata y G. ovalis representan conceptos específicos que han permanecido relativamente
Tabla 4. Musgos del estado de Aguascalientes colectados por Delgadillo y Peña en 2013. Los nombres precedidos por un asterisco fueron citados por Sharp et al. (1994). La columna de la derecha indica el número de celdas en las cuales se han registrado las especies colectadas en Aguascalientes.

Aloina hamulus (Müll.Hal.) Broth.

Anomobryum filiforme (Dicks.) Solms. in Rabenh.

Barbula orizabensis Müll. Hal. 2

Brachymenium mexicanum Mont.

Brachymenium systylium (Müll.Hal.) A.Jaeger

Braunia andrieuxii Lorentz

* Braunia secunda (Hook.) Bruch \& Schimp.

Bryum argenteum Hedw.

Bryum billarderi Schwägr.

Bryum chryseum Mitt.

*Campyliadelphus chrysophyllus (Brid.) Kanda

Campylopus pilifer Brid.

*Ceratodon purpureus (Hedw.) Brid. ssp. stenocarpus

(Bruch \& Schimp.) Dixon

Crossidium crassinervium (De Not.) Jur.

Desmatodon convolutus (Brid.) Grout

Didymodon australasiae (Hook. \& Grev.) R.H.Zander

Didymodon revolutus (Cardot) R.S.Williams

Didymodon rigidulus Hedw. var. gracilis (Schleich. ex

Hook. \& Grev.) R.H.Zander

Didymodon rigidulus Hedw. var. icmadophilus (Schimp.

ex Müll. Hal.) R.H.Zander

Didymodon rigidulus Hedw. var. rigidulus

Entosthodon apiculatopilosus (Cardot) Fife

Erythrodontium longisetum (Hook.) Paris

Erythrodontium squarrosum (Müll. Hal.) Paris

Fabronia ciliaris (Brid.) Brid. var. polycarpa (Hook.) W.R.Buck

Fabronia macroblepharis Schwägr.

Fissidens bryoides Hedw.

Fissidens crispus Mont.

Fissidens guianensis Mont.

Fissidens sublimbatus Grout

*Grimmia elongata Kaulf.

*Grimmia laevigata (Brid.) Brid.

Grimmia longirostris Hook.

*Grimmia ovalis (Hedw.) Lindb.

Grimmia pulla Cardot

Hennediella heteroloma (Cardot) R.H.Zander

Jaffueliobryum arsenei (Thér.) Thér.

Leptodontium flexifolium (Dicks. ex With.) Hampe

Leskea angustata Taylor

Pleuridium mexicanum Cardot

Pogonatum campylocarpum (Müll. Hal.) Mitt.

Pogonatum oligodus (Müll. Hal.) Mitt.

Pseudocrossidium replicatum (Taylor) R.H.Zander

Syntrichia chisosa (Magill, Delgad. \& L.R.Stark) R.H.Zander

Syntrichia fragilis (Taylor) Ochyra

Tortula acaulon (With.) R.H.Zander

estables, pero se necesita confirmar su existencia como parte de la flora estatal a través de la revisión de los ejemplares originales o de la recolección de otras muestras. Grimmia longirostris también fue citado por Sharp et al. (1994). 


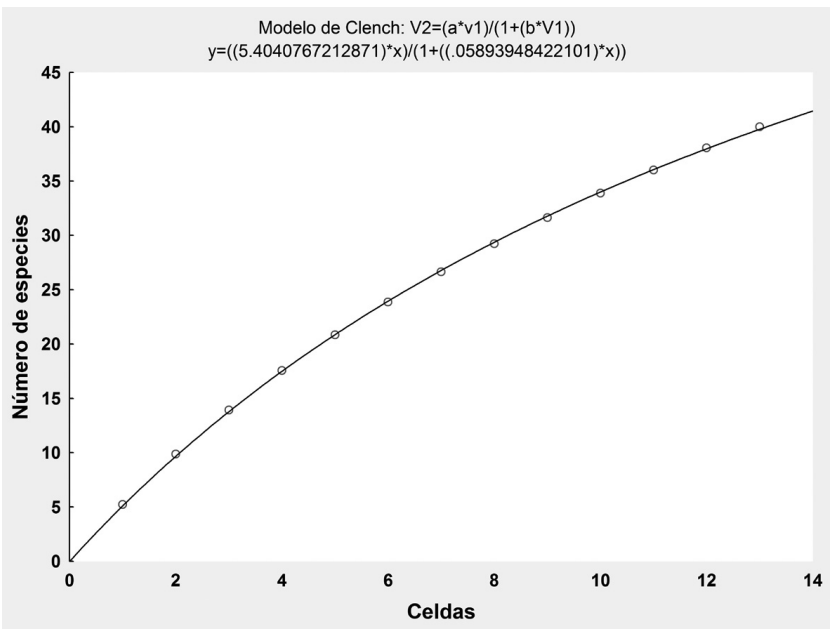

Figura 3. Curva de acumulación de especies de musgos en el estado de Aguascalientes. Los círculos representan las unidades de muestreo (celdas de 6 minutos de arco por lado). La asíntota se alcanza a las 91 especies, lo que indica un nivel de completitud de $43 \%$ de los musgos en el estado (39 especies con registros fehacientes). Los parámetros de la curva se indican en la ecuación de la parte superior de la figura.

Con respecto al listado del Tabla 4, algunas especies como Aloina hamulus, Didymodon revolutus, Leskea angustata y Pseudocrossidium replicatum son frecuentes; las dos primeras y la última crecen sobre rocas calcáreas, mientras que la tercera se encuentra generalmente como epífita. En contraste, Crossidium crassinervium, Pleuridium mexicanum y Tortula acaulon están mal representados en la muestra, probablemente porque su pequeña estatura o porque su condición efímera los hacen poco notables en el campo. No obstante, los tres taxa son de interés por sus modificaciones estructurales; $C$. crassinervium lleva filamentos fotosintéticos sobre la costa mientras que los dos últimos tienen cápsulas cleistocárpicas.

Las poblaciones de Campylopus pilifer tienen variaciones morfológicas importantes con respecto a las descritas en manuales florísticos (p.ej., Sharp et al. 1994) y podrían confundir su determinación. Las hojas son más estrechas y el pelo apical está ausente en la mayoría de las muestras, pero la estructura foliar, incluyendo dos hileras de leucocistos y lamelas abaxiales bajas en la costa, identifican claramente a esta especie. Por su parte, en los ejemplares de Didymodon australasiae, aunque otros caracteres diagnósticos están presentes, las células basales de la hoja no están claramente diferenciadas de otras células foliares y el ápice de la hoja no es subcuculado. En algunos ejemplares de Pseudocrossidium replicatum, la espiral de la lámina de la hoja está poco desarrollada.

Desde el punto de vista de su distribución, las muestras incluyen ejemplares de Grimmia pulla, Hennediella heteroloma y Jaffueliobryum arsenei. Las tres son endémicas a México; G. pulla está ampliamente distribuida a lo largo del
Eje Neovolcánico, mientras que $H$. heteroloma se extiende desde Chihuahua hasta Morelos y la última, desde cerca de la ciudad de Zacatecas hasta el sur de Querétaro.

Esfuerzo de colecta. La evaluación del esfuerzo de colecta permite suponer que en Aguascalientes existen unas 91 especies de musgos, tal y como lo revela el valor que alcanza la asíntota de la curva de acumulación (Figura 3). De ese número se ha documentado la presencia de 39 especies, o sea, un nivel de completitud de $43 \%$.

Riqueza conocida. La riqueza conocida hasta la fecha (39 especies) no se distribuye de manera homogénea en el estado de Aguascalientes; las 15 localidades que la base de datos reporta alojan los 109 registros de las 39 especies (Figura 1). De hecho, solamente 13 celdas, de un total de 70 en que se dividió al estado, registran especies de musgos según especímenes herborizados; en las otras 57 celdas no hay registro de especies (Figura 2). Las dos celdas que registran la mayor riqueza (11especies, Figura 2, Tabla 2) se encuentran sobre la Sierra Madre Occidental, en la Sierra Fría (celda 18) y la Sierra del Laurel (celda 59).

En el Tabla 4 se indica el número de celdas donde se ha registrado cada especie. La especie más ampliamente distribuida es Grimmia longirostris, registrada en ocho celdas; en segundo lugar se ubica Didymodon revolutus (5 celdas). Por

Tabla 5. Especies conocidas de Jalisco y Zacatecas, según fuentes bibliográficas, cuyo modelo de nicho ecológico proyecta superficie de ocupación en el estado de Aguascalientes.

\section{Especie}

1. Anoectangium aestivum (Hedw.) Mitt.

2. Bartramia microstoma Mitt.

3. Bryoerythrophyllum campylocarpum (Müll.Hal.) Crum

4. Bryoerythrophyllum recurvirostrum (Hedw.) Chen

5. Campylophyllum sommerfeltii (Myr.) Hedenäs

6. Campylopus nivalis (Brid.) Brid.

7. Campylopus oblongus Thér.

8. Campylopus tallulensis Sull. \& Lesq.

9. Cyrto-hypnum mexicanum (Mitt.) W.R.Buck \& H.A.Crum

10. Entosthodon obtusifolius Hook.

11. Fabronia ciliaris (Brid.) Brid.

12. Fissidens asplenioides Hedw.

13. Funaria hygrometrica Hedw.

14. Grimmia pilifera P. Beauv.

15. Orthotrichum pycnophyllum Schimp. ex Müll. Hal.

16. Platygyriella densa (Hook.) W.R.Buck

17. Plaubelia sprengelii (Schwägr.) R.H.Zander var. stomatodonta

(Cardot) R. H. Zander

18. Pohlia elongata Hedw.

19. Rhexophyllum subnigrum (Mitt.) Hilp.

20. Rhynchostegium riparioides (Hedw.) Cardot

21. Syntrichia amphidiacea (Müll. Hal.) R.H.Zander

22. Timmiella anomala (B.S.G.) Limpr.

23. Trichostomum brachydontium Bruch

24. Trichostomum tenuirostre (Hook. \& Taylor) Lindb.

25. Weissia controversa Hedw. 

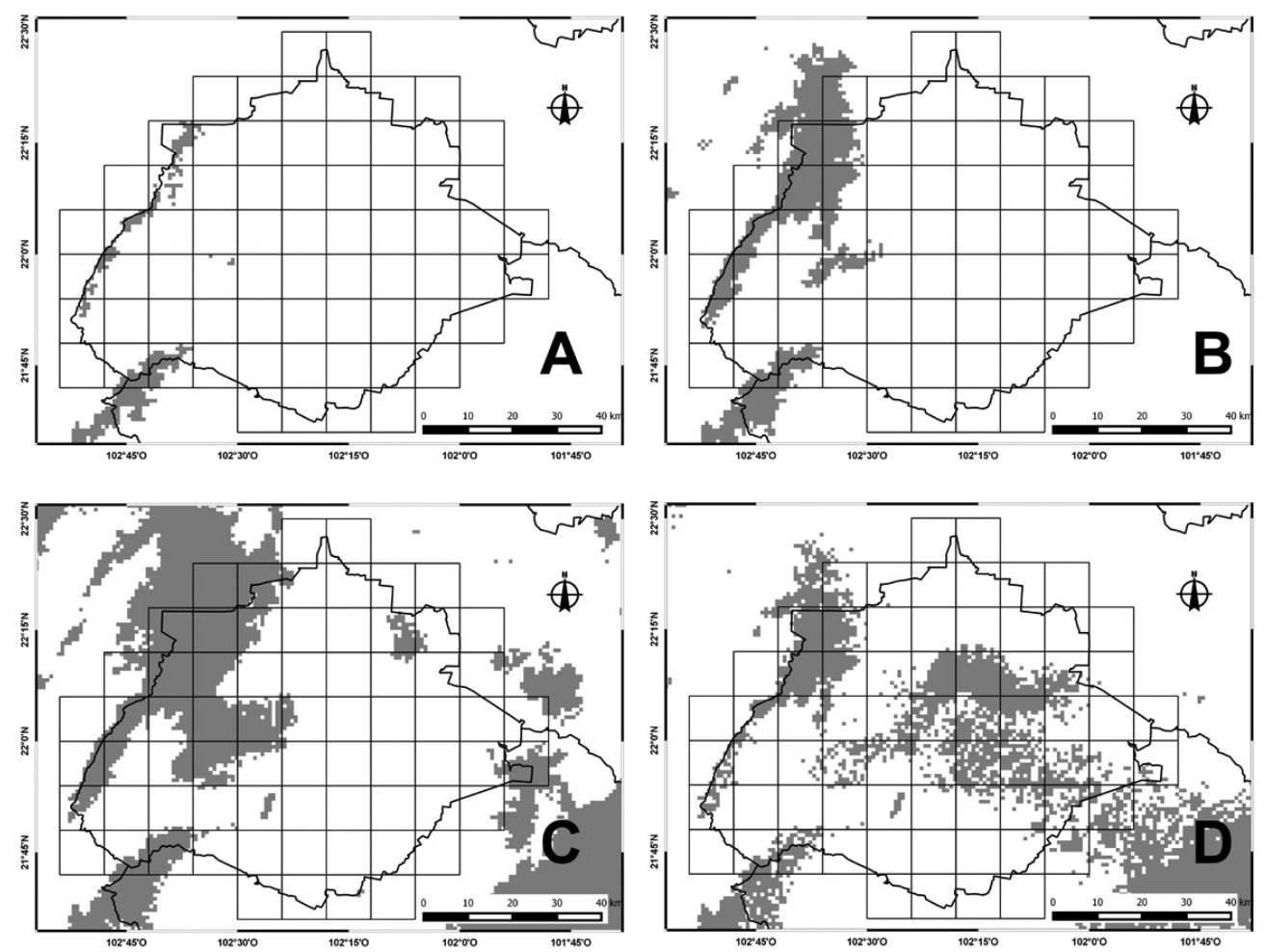

Figura 4. Modelo de nicho ecológico de (A) Anoectangium aestivum (Hedw.) Mitt., (B) Bryoerythrophyllum campylocarpum (Müll. Hal.) Crum, (C) Grimmia pilifera P. Beauv. y (D) Orthotrichum pycnophyllum Schimp. ex Müll. Hal. proyectados hacia Aguascalientes.

otra parte, 23 son las especies menos frecuentes, conocidas hasta la fecha solamente de una celda.

Riqueza estimada. De las 41 especies presentes en Jalisco y Zacatecas con modelo elaborado, solamente 25 de ellas proyectan su distribución hacia el estado de Aguascalientes (Tabla 5). A manera de ejemplo, se ilustran los modelos de distribución potencial de cuatro de ellas (Figura 4).

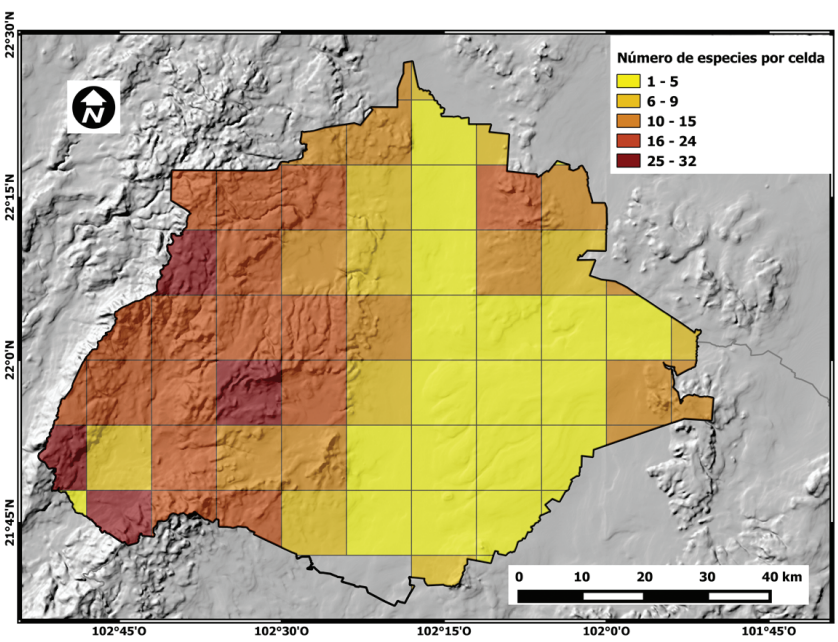

Figura 5. Riqueza estimada de musgos en el estado de Aguascalientes. Los tonos indican el número de especies estimadas en cada celda $\left(6^{\prime} \times 6^{\prime}\right)$ a partir de los registros conocidos de 39 especies en el estado y de los modelos de distribución potencial de 25 especies presentes en Jalisco y Zacatecas.
La distribución conocida de las 39 especies conocidas de Aguascalientes, complementada con el área potencial de las 25 especies presentes sólo en Jalisco y Zacatecas, permitió generar el mapa de riqueza mostrado en la Figura 5. El escenario mostrado en dicha figura sugiere que en todo el estado de Aguascalientes podrían registrarse especies de musgos, con una mayor preponderancia hacia su región occidental.

Los modelos de nicho ecológico predicen que la mayor riqueza de musgos se concentra en las celdas 18 y 59 , con 32 y 31 especies respectivamente, ubicados en las porciones serranas del occidente del estado (Tabla 2, Figura 5), mientras que los valores menores de riqueza se registran en sus valles, como el Valle de Aguascalientes, el Valle de Calvillo y la región de El Llano, que tienen de una a ocho especies. Los modelos no lograron subsanar las lagunas de información en dos celdas, 67 y 68; tales celdas se encuentran en la zona de baja riqueza florística de musgos, por lo que es muy posible que las especies presentes allí no sean numerosas.

Como se ha indicado, la mayor concentración de especies se observa en la porción occidental del estado, que corresponde a las porciones de la Sierra Madre Occidental. La riqueza potencial sugiere que gran parte de esa sierra en Aguascalientes debería contener valores más o menos equivalentes de riqueza de especies congruentes con la principal afinidad ecológica de los musgos por ambientes montañosos y templados, característicos de esa cadena montañosa en el país. 


\section{Discusión}

Aguascalientes es un estado comparativamente pequeño, con un relieve en el que destaca la zona montañosa del oeste y dos elevaciones que alcanzan los 3,000 m (Cerro de la Ardilla y el Cerro del Laurel). Su territorio ha sido fraccionado principalmente para la ganadería y la agricultura y la vegetación original ha sido fuertemente perturbada; la observación rápida de los matorrales y los encinares visitados hasta ahora parece indicar que existen pocos hábitats para los musgos. En algunos sitios protegidos existe mayor diversidad de musgos, pero los sitios abiertos, particularmente aquellos con sustratos calizos, están ocupados por pocas especies. Además de la influencia humana, la baja humedad relativa, la baja precipitación anual y la alta insolación, son aparentemente limitantes para la diversidad de los musgos en el estado de Aguascalientes. De acuerdo con datos del Servicio Meteorológico Nacional (2014), se puede sugerir que los valores de la temperatura y la precipitación para el estado son, en general, propios de un área semiseca y, por tanto, se esperaría un contingente bajo de especies de musgos. No obstante, en las elevaciones más altas con un clima templado en los que existen bosques de pino, encino y cedro, se puede esperar un número mayor de musgos.

Los análisis parecen dar sustento a las observaciones antes mencionadas. El número comparativamente bajo de especies representadas en nuestro muestreo, aunado a las que se citan en la literatura, apenas alcanza 45 especies. La curva de acumulación de especies indica que se pueden esperar hasta 91 especies para el estado (Figura 3 ) y aunque se deben visitar muchas localidades todavía (cf. Figura 1), es evidente que la riqueza de especies de musgos de Aguascalientes sólo es comparable a la de partes de los dos estados que lo rodean (Figuras 2, 5). La riqueza estimada para las celdas de la mitad occidental del estado es mayor que la de otros sectores, pero no rebasa las 32 especies por celda (Figura 5). En ese sentido, la distribución de las especies en Aguascalientes podría interpretarse como una extensión de la flora de musgos de Zacatecas y de Jalisco pues la mayoría de las especies ha sido registrada para esos dos estados.

Además del número comparativamente bajo de especies conocidas para la flora de musgos de Aguascalientes, se debe mencionar que la variación morfológica de algunas especies no es privativa de la flora de Aguascalientes y que se desconoce si depende de algún parámetro ecológico local. El borde poco enrollado en ciertas muestras de Pseudocrossidium replicatum, las hojas más estrechas y casi desprovistas de pelo apical en Campylopus pilifer y las células foliares proximales pobremente diferenciadas de Didymodon australasiae, podrían ser debidas a insuficiente humedad y perturbación durante el periodo de crecimiento. Por su parte, los filamentos foliares de Crossidium crassinervium o las cápsulas cleistocárpicas de Pleuridium mexicanum y Tortula acaulon representan adaptaciones de cada especie en su área de distribución. During (1992), en una síntesis sobre las estrategias de las plantas en ambientes hostiles, alude a ideas sobre el crecimiento y la supervivencia determinados por estrés y por perturbación. La falta de agua y minerales o la destrucción inducida por sequía, erosión edáfica o fuego, inciden sobre el nivel de productos fotosintéticos. Tales presiones son parte del ambiente en Aguascalientes y podrían dar lugar a las modificaciones estructurales de los musgos. No obstante, no existe evidencia de la respuesta de las plantas locales a factores como sequía y perturbación, aunque hay numerosos estudios sobre la tolerancia de las briofitas a la desecación (cf. Proctor et al., 2007).

Los modelos de nicho ecológico de la figura 4 muestran la distribución potencial de especies conocidas de Jalisco y Zacatecas que podrían esperarse en varias partes de Aguascalientes, es decir, se debe esperar una mayor correspondencia de las floras de los tres estados con la mejor exploración del sector noroccidental, particularmente en los límites con Zacatecas cuya vegetación arbórea debe albergar un mayor número de musgos. Naturalmente, también debe mencionarse la reducida extensión geográfica y el clima, como factores limitantes de la diversidad de musgos en Aguascalientes. La completitud de los catálogos, la distribución local y mundial de la flora serán revisadas con los resultados de nuevos trabajos de campo. La celeridad y la continuidad de estos trabajos son urgentes pues los desarrollos agrícolas, ganaderos, industriales y el crecimiento de las poblaciones humanas ponen en peligro la existencia de pequeños enclaves donde pueden persistir muchas especies.

\section{Agradecimientos}

A Ana Susana Estrada por la generación de los modelos de nicho ecológico y el manejo de la base de datos. Dos revisores anónimos contribuyeron con su opinión a la mejor comprensión de los resultados de esta investigación.

\section{Literatura citada}

Colwell R.K. 2009. EstimateS: Statistical estimation of species richness and shared species from samples Version 8.2.0. Disponible en linea <http://viceroy.eeb.uconn.edu/estimates>. (Consultado: agosto 2014)

Colwell R.K. y Coddington J.A. 1994. Estimating terrestrial biodiversity through extrapolation. Philosophical Transactions of the Royal Society (Series B) 345:101-118.

Crum H.A. 1951. The Appalachian-Ozarkian element in the moss flora of Mexico with a check-list of all known Mexican mosses. Ph. D. Dissertation. University of Michigan. Ann Arbor. 504 pp.

Cruz-Cárdenas G., Villaseñor J. L., López-Mata L. y Ortiz E. 2012. Potential distribution of Humid Mountain Forest in Mexico. Botanical Sciences 90:331-340.

Delgadillo-Moya C. 2010. LATMOSS 2010. <http://www.ibiologia.unam.mx/briologia/www/index/Bases.html> (consultado: agosto 2014) 
Delgadillo-M C. y Cárdenas-S M.A. 1979. Musgos de Zacatecas, México. I. Boletín de la Sociedad Botánica de México 38:1-6.

Delgadillo-M C. y Cárdenas-S A. 1987. Musgos de Zacatecas, México. III. Síntesis y fitogeografía. Boletín de la Sociedad Botánica de México 47:13-24.

Delgadillo M.C. y Cárdenas S.A. 1996. A preliminary checklist of the mosses of Guanajuato, Mexico. Flora del Bajío y Regiones Adyacentes, Fasc. complementario XI: 1-14.

De Luna E. y Gómez-Velasco G. 2008. Morphometrics and the identification of Braunia andrieuxii and B. secunda (Hedwigiaceae, Bryopsida). Systematic Botany 33:219-228.

During, H.J. 1992. Ecological classifications of bryophytes and lichens. En: Bates, J.W. y Farmer A.M. Eds. Bryophytes and Lichens in a Changing Environment, pp. 1-31, Clarendon Press, Oxford.

Gotelli N.J. y Colwell R.K. 2001. Quantifying biodiversity: procedures and pitfalls in the measurement and comparison of species richness. Ecological Letters 4:379-391.

Herrera-Paniagua P., Delgadillo-M. C., Villaseñor-Ríos J.L. y Luna-Vega I. 2008. Floristics and biogeography of the mosses of the state of Querétaro, Mexico. The Bryologist 111:41-56.

Hijmans R.J., Cameron S.E., Parra J.L., Jones P.G. y Jarvis A. 2005. Very high resolution interpolated climate surfaces for global land areas. International Journal of Climatology 25:19651978.

IUCN [International Union for Conservation of Nature]. 2001. IUCN Red List Categories and Criteria: Version 3.1. IUCN. Species Survival Commission. IUCN, Gland, Switzerland and Cambridge.

Jiménez-Valverde A. y Hortal J. 2003. Las curvas de acumulación de especies y la necesidad de evaluar la calidad de los inventarios biológicos. Revista Ibérica de Aracnología 8:151-161.

Liu C., White M. y Newell G. 2013. Selecting thresholds for the prediction of species occurrence with presence-only data. Journal of Biogeography 40:778-789.

López-Mata L., Villaseñor J.L., Cruz-Cárdenas G., Ortiz E. y Ortiz-Solorio C. 2012. Predictores ambientales de la riqueza de especies de plantas del Bosque Húmedo de Montaña en México. Botanical Sciences 90:27-36.

Moat J. 2007. Conservation assessment tools, extension for Arc View 3.x, version 1.2. GIS Unit, Royal Botanic Gardens, Kew, Londres.

Newbold T. 2010. Applications and limitations of museum data for conservation and ecology, with particular attention to species distribution models. Progress in Physical Geography 34:3-22.

Peterson A.T., Soberón J., Pearson R.G., Anderson R.P., MartínezMeyer E., Nakamura M. y Araújo M.B. 2011. Ecological Niches and Geographic Distributions. Princeton University Press, Princeton.

Phillips S., Anderson R. y Schapire R. 2006. Maximum entropy modeling of species geographic distributions. Ecological Modelling 190:231-259.

Phillips S.J. y Dudik M. 2008. Modeling of species distributions with MaxEnt: new extensions and a comprehensive evaluation. Ecography 31:161-175.

Proctor, M.C., M.J. Oliver, A.J. Wood, P. Alpert, L.R. Stark, N.L. Cleavitt y B.D. Mishler. 2007. Desiccation-tolerance in bryophytes: a review. Bryologist 110:595-621.

Servicio Meteorológico Nacional - Conagua. Aguascalientes. 2014. <http://smn.cna.gob.mx/index.php?option=com_conte nt\&view=article\&id=159:aguascalientes\&catid=14: normalespor-estacion> (Consultado: Enero 9, 2014).

Sharp A.J., Crum H.A. y Eckel P.M. Eds. 1994. The Moss Flora of Mexico. Memoirs of the New York Botanical Garden 69: i+xvii, 1-1113.

Soberón J. y Llorente J.B. 1993. The use of species accumulation functions for the prediction of species richness. Conservation Biology 7:480-488.

StatSoft, Inc. 2011. STATISTICA (data analysis software system), version 10. <www.statsoft.com>

Suárez-Mota M.E. y Villaseñor J.L. 2011. Las Compuestas endémicas de Oaxaca, México: diversidad y distribución. Boletín de la Sociedad Botánica de México 88:55-66.

Recibido: 2 de agosto de 2014

Aceptado: 14 de septiembre de 2014 